\title{
An Artificial Neural Network Based Methodology for the Prediction of Power \& Torque Coefficients of a Two Bladed Airfoil Shaped H-Rotor
}

\author{
Agnimitra Biswas ${ }^{1, *}$ and R. Gupta ${ }^{2}$ \\ ${ }^{I}$ PhD Scholar, Deptt. of Mechanical Engg., NIT Silchar, Assam (India) \\ ${ }^{2}$ Department of Mechanical Engineering, NIT Silchar, Assam (India)
}

\begin{abstract}
An artificial neural network based model can effectively predict any functional relationship. In this paper, a neural network model is used to predict power coefficient and torque coefficient of a two bladed airfoil shaped H-rotor as function of different input parameters. The important input parameters considered are blade tip speed, free stream velocity with blockage and rotor inlet velocity. The values of all the process parameters are taken from the experimental work done on two-bladed airfoil shaped H-rotor. The rotor was earlier designed, fabricated, and tested in a subsonic wind tunnel available in the department. Since neural networks are good at interpolation, once the model is properly trained \& tested, it has successfully interpolated the values of power and torque coefficients within an acceptable accuracy. Initially, the optimum no. of neurons in the hidden layer has been found out using hit and trial method by training the network using back propagation learning algorithm. The effect of increasing the size of training and testing data set is studied as well. It is found that only a single neuron has been able to predict both the coefficients successfully. A strategy has been developed to reduce both the training and testing errors. The root mean squared functional errors (rms error) of testing and training for power coefficient prediction are 0.0357 and 0.0387 respectively, while the corresponding values for torque coefficients are 0.0283 and 0.0449 respectively. The proposed methodology is fast and accurate. And testing error being less than the training error, makes the proposed algorithm a superior one.
\end{abstract}

Keywords: Two-bladed airfoil shaped H-rotor, power coefficient, torque coefficient, functional rms error.

\section{INTRODUCTION}

The performance of a neural network model is dependent on many parameters, like size of the training and testing data, number of neurons in the hidden layer, number of hidden layers, processing functions on the neurons, time required to determine the best architecture, and accuracy of prediction. The size of the data- set is decided by the performance of the network, while optimum number of hidden neurons should be obtained from training the network. Otherwise, desired performance may not be obtained as too much increase of hidden neurons will unnecessarily reduce the testing error though training error may be improved [1]. The optimum number of hidden layer neurons is obtained by hit and trial method by running the code for number of times with different training and testing sets and checking the prediction errors. The point, when the prediction error is dropped below an acceptable value, the run is stopped; and the number of hidden neurons of that particular network architecture is stored for further simulation. The target in designing the model is to reduce both the training and testing errors simultaneously. The network is termed to be a superior one if more testing dataset fit the network that predicts the functional relationship between input and output, and the testing error comes out to be less than the training

*Address correspondence to this author at the NIT Silchar (Assam) India, 788004; Tel: +91 3842 242913; Fax: +91 3842 233797;

E-mail:agnibis@yahoo.co.in error. It is seen from the literature that the functional relationship between three inputs and single output system can be modeled using artificial neural network and that too, with minimum number of training and testing datasets [1]. Too many hidden layers prolong the computational time, though a single hidden layer can accurately predict for such system. As the cost involved in data generation is high, and also the time consumed for computer simulation is more, in this paper it has been decided to develop a strategy to predict the outputs with minimum number of datasets. For this, the network is fitted with a few initial values, and more data are added seeing the prediction error. The data for training and testing of the network are taken from the experimental results of the rotor, which was tested in an open circuit subsonic wind tunnel in the department [2]. The strategy adopted here is to reduce both the training and testing errors. The smaller the testing error between target power coefficient (and also torque coefficient) and its predicted value, the more effective the neural network will become. In this paper, root mean squared functional error is considered to be the basis to evaluate effectiveness of the network.

\subsection{Scope of Neural Network Modeling in Renewable Energy Systems}

Generalized Artificial Neural Network (ANN) models for any physical system have the capability of processing parallel information fast and accurately. The Renewable energy systems like wind energy systems have many wind related information that flow into the system. There are important wind properties such as wind speed at the rotor inlet, 


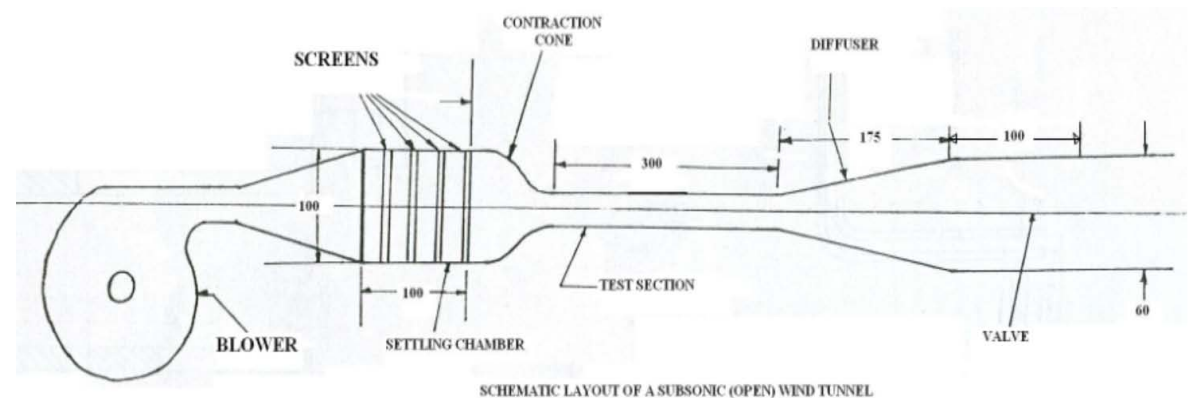

(ALL DMENSIONS ARE IN mn.)

Fig. (1). Schematic layout of open circuit subsonic wind tunnel.

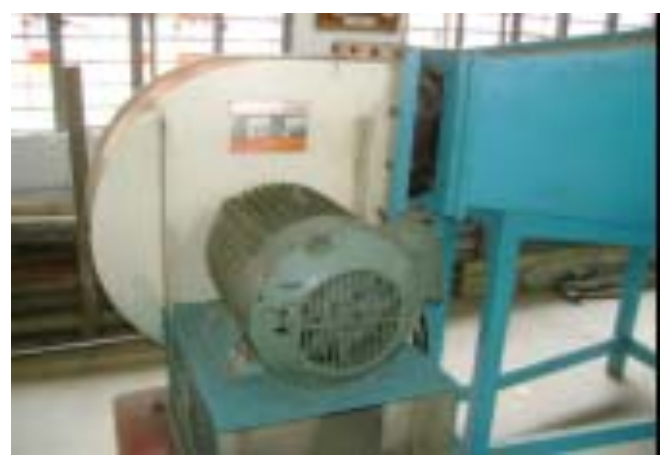

(a)

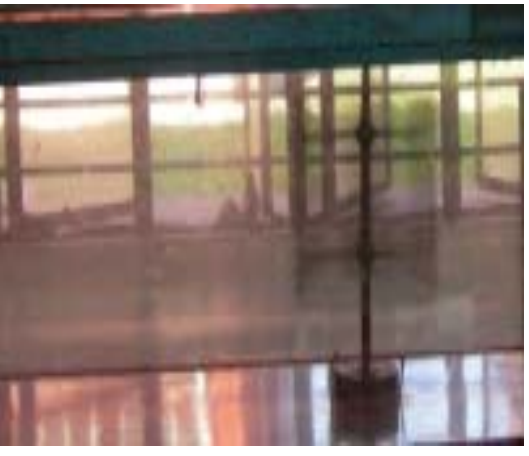

(b)

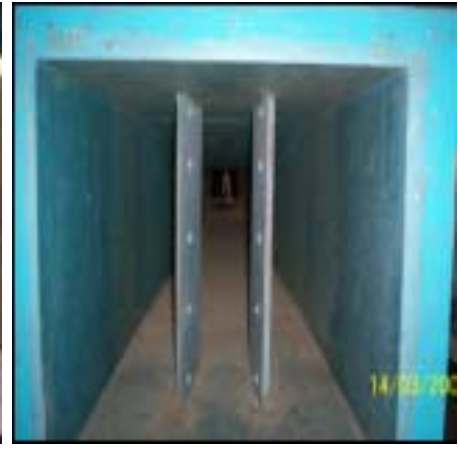

(c)

Fig. (2). (a). The centrifugal blower section. (b) The test section with the rotor. (c) The end valve.

free stream wind speed, blade tip speed etc. that directly control the performance coefficients of the wind turbine, i.e. power coefficient and torque coefficient. It is necessary to accurately predict the performance coefficients to maximize the effectiveness of the investments made on such system. Like many other disciplines, ANN can be used in wind based renewable systems because of its accuracy. There are comprehensive reviews of ANN modeling available for any general energy systems [3] and also for renewable energy systems in particular [4]. Kalogirou [4] applied ANN to predict wind speed of a small wind generator; in addition to his works on other Renewable energy systems, e.g., solar steam generator, solar water heating systems, photovoltaic systems etc. Apart from this, ANN is also applied as a classification mechanism for determining average wind speed and power [5].

\section{EXPERIMENTAL SET-UP FOR THE TWO BLADED AIRFOIL SHAPED H-ROTOR}

The data for training and testing of the network are taken from the experimental results of the rotor. For carrying out the experiments, an open circuit subsonic wind tunnel was utilized, available in the department. The schematic layout of the wind tunnel is shown in Fig. (1). The blower section consists of one axial flow fan having variable pitch blades placed in cylindrical casing, which is driven by a three phase $15 \mathrm{~kW}(20 \mathrm{hp})$ motor having rated $2890 \mathrm{rpm}$ as shown in Fig. (2a). In the wind tunnel, the cross-section of the test section is $30 \mathrm{~cm} \times 30 \mathrm{~cm}$, and the length is $300 \mathrm{~cm}$. The test section with the rotor inside is shown in Fig. (2b). The operating range of the wind tunnel is between 0 and 30 $\mathrm{m} / \mathrm{sec}$. The turbulence intensity in the wind tunnel is neglected since it is negligible for the present case. The details of the wind tunnel can be found in the literature [6]. The cross section of the blades of the two-bladed airfoil shaped H-rotor was built into airfoil shape. The height of the rotor was $20 \mathrm{~cm}$ and the blades were $5 \mathrm{~cm}$ wide as shown in Fig. (3). The material used for the blades was aluminum. The central shaft was made up of mild-steel of $16 \mathrm{~mm}$ diameter. The supports, i.e. the struts, for holding the blades in place were mild steel bolts of $5 \mathrm{~mm}$ diameter and $15 \mathrm{~cm}$ length. The diameter of the rotor was changed by changing the position of nuts on the bolts, while height of the rotor was kept fixed at $20 \mathrm{~cm}$. Eleven height-to-diameter ratios were created. The blade tip speeds and rotor inlet velocities for each of

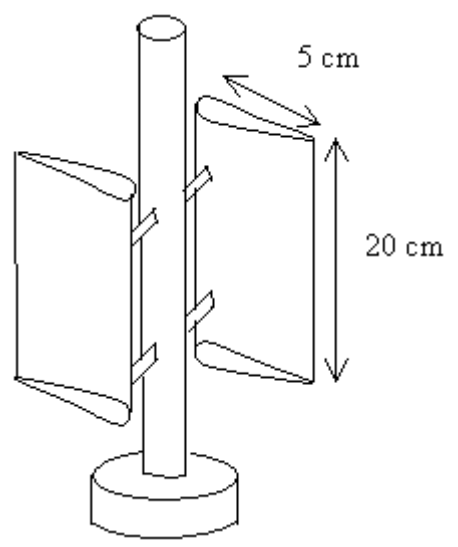

Fig. (3). Schematic view of two bladed airfoil shaped H-rotor. 


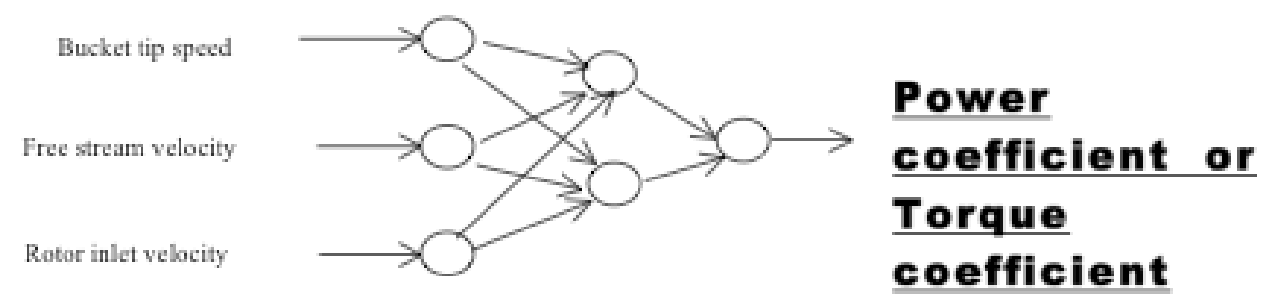

Fig. (4). A typical neural network architecture.

those eleven height-to-diameter ratios were obtained by varying the position of the valve at the end of the tunnel (Fig. 2c) five times, which was adjustable between $3 \mathrm{~cm}$ to $25 \mathrm{~cm}$. The rotor rpm was measured using a digital tachometer having a least count of $1 \mathrm{rpm}$, and the rotor inlet velocity was measured using Pitots static tube. The values of free stream velocity were obtained by the same procedure without placing the rotor model in the test section. A total of 55 datasets for the input and output parameters were obtained from the experiments [2].

\section{METHODOLOGY IN THE PREDICTION OF POWER COEFFICIENTS \& TORQUE COEFFI- CIENTS}

A feed forward artificial neural network trained using back-propagation learning algorithm [3] is utilized. The Levenberg-Marquardt back propagation algorithm of Matlab has been used in the present case. The process parameters considered are blade tip speed, free stream velocity with blockage and rotor inlet velocity. Thus, the input layer of the neural network consists of three neurons, while the output layer consists of a single neuron that corresponds to power coefficient and torque coefficient at a time. A typical architecture with three neurons in input layer, two neurons in hidden layer, and one neuron in output layer is shown in the Fig. (4). The optimum number of neurons in the hidden layer is decided by hit and trial approach so that the error in prediction is minimized.

\subsection{Normalization}

The numerical values of different process parameters lie in different ranges. The three input parameters i.e. blade tip speed, free stream velocity with blockage and rotor inlet velocity are normalized such that their values lie between 0.1 and 0.9. This is in accordance with the literature [1].

\subsection{Selection of the Number of Hidden Layers}

Earlier studies did not indicate any improvements of multiple hidden layers over single hidden layer systems. The theorem by white [7] states that one hidden layer is sufficient to map any non-linear functional relationship with a reasonable level of accuracy. Moreover, too many hidden layers increase the computational time without increasing the accuracy much. So, only a single hidden layer is used.

\subsection{No of Neurons in Hidden Layer}

It is necessary to select optimum number of neurons in order that the effective error in both training and testing drops to an acceptable value. Lawrence and Petterson [8] provided a scheme to determine the number of neurons by the following expressions:
Minimum number of training data $=2 \times$ (input + hidden + output) neurons

Maximum number of training data $=10 \mathrm{x}$ (input + hidden + output) neurons

\subsection{Selection of Processing or Transfer Functions}

There are many inbuilt processing functions (or transfer functions) of Matlab software, like logarithmic sigmoid function (logsig), tangential sigmoid function (tansig), pure linear function (purelin) etc. From the earlier works [9], it is observed that both logsig and tansig produce almost the same performance. Considering this, only logsig processing function has been used.

\subsection{Training and Testing Error}

The training or the testing error of an artificial neural network can be expressed in the form of functional root mean square (rms) error. These are given in $\mathrm{eq}^{\mathrm{n}} \mathrm{s} 3 \& 4$. A neural network is said to be an effective one when both training and testing errors are minimized.

$$
\begin{aligned}
& \mathrm{RMS}_{\text {error }}^{\text {training }}=\sqrt{\sum \frac{\left(\text { target }- \text { trained }^{2}\right.}{n * \text { target }^{2}}} \\
& \mathrm{RMS}_{\text {error }}^{\text {testing }}=\sqrt{\sum \frac{\left(\text { target }- \text { predicted }^{2}\right.}{n * \text { target }^{2}}}
\end{aligned}
$$

Where, ' $n$ ' is the number of training / testing dataset. Target is the measured values of power coefficient and torque coefficient whose sizes are different in their respective training and testing sets.

\subsection{Increase of the Size of Training and Testing Data Set}

If the desired accuracy, i.e. functional root mean squared (rms) error in training and testing have been minimized (below an acceptable limit), is not attained, then the sizes of data sets are changed. The strategy is: transfer a test data showing maximum deviation to the training dataset for which the functional rms error is not acceptable. And in lieu of it, create two additional data sets in the testing set in a random manner. In that manner, the sizes of the training and testing data sets are increased. With the new data sets, the network is fitted again. The process is continued till the desired accuracy is achieved.

\subsection{Selection of the Best Network Configuration}

The entire methodology followed to select the best network topology is illustrated in the form of a flow chart as shown in Fig. (5). The proposed methodology is used to predict for power coefficient and torque coefficient of the rotor. 
All the data are normalized between 0.1 and 0.9 to produce uniformity in values. The initial size of the training detaset is taken to be 10; obtained from equation 1, by taking a single neuron in the hidden layer. Each dataset corresponds to a unique combination of blade tip speed, free stream velocity with blockage, rotor inlet velocity, power coefficient, and torque coefficient as obtained from the experiments. Half the size of training detaset is taken as the size of testing dataset, random wise, at the start. The dataset in the testing detaset are such that the network has never seen them while training. With this, in the first stage, the Matlab code is run for as many epochs for which the minimum gradient in the back propagation learning is reached. The inbuilt 'trainlm' back propagation learning function of Matlab is utilized for the purpose. The number of hidden layer neurons is increased from one to a maximum of six. For the selected seven network topologies, at the end of their respective runs with the same training and testing datasets, the training and testing errors are compared. The number of hidden layer neurons of that network topology which has produced lowest training and testing errors compared to the rest are determined. If both the rms errors ( $\mathrm{eq}^{\mathrm{n}} \mathrm{s} 3 \& 4$ ) are minimized to the satisfaction, and the rms error in testing is lower than that in testing, then the best fitted network is obtained; else, a test data having maximum deviation is transferred to the training dataset, and two fresh datasets are created testing. In the second stage with new training and testing datasets, again the best network topology is searched. This time, only three ar- chitectures are searched: the network with the optimum number of neurons as obtained from the first stage, one less and one more neuron in the hidden layer. Again, best network topology is selected out of the three, based on the lowest errors. Again datasets are shifted and added. In this way, the sizes of training and testing datasets are increased until the desired accuracy is attained and all the data are fitted.

\section{RESULTS \& DISCUSSION}

In the present work, the proposed algorithm is used to predict the power and torque coefficients of a two bladed airfoil shaped H-rotor based on the experimental values of the process parameters. Separate artificial neural networks are fitted for power coefficient and torque coefficient predictions. From the results, it is observed that, in both the cases, the network configuration is the same, i.e., the number of neurons in the hidden layer is only one. A maximum of 5000 epochs has been allowed in each run. The objective is to fit all the 55 data of each parameter. It has been decided that functional root mean squared error (rms error) in both training and testing should be within 5\%. For the prediction of both the outputs, 35 data are used to train the network, and 20 validation i.e. test data are used to asses the performance of the fitted networks. The training and testing data utilized in both the predictions are shown in Table A1-A4 (incorporated in Appendix A). The test data are selected in such a way that they have covered the whole range of the parameters.

Table 1. Effect of Training and Testing Dataset on Rms Functional Error for Power Coefficient Prediction

\begin{tabular}{|c|c|c|c|}
\hline \multicolumn{2}{|c|}{ Number of Dataset } & \multicolumn{2}{c|}{ Functional Rms Error in Prediction } \\
\hline \hline Training Data & Testing Data & Training Error & Testing Error \\
\hline 10 & 5 & 0.0294 & 0.0589 \\
\hline 15 & 10 & 0.0045 & 0.0778 \\
\hline 20 & 13 & 0.0116 & 0.0534 \\
\hline 25 & 18 & 0.0269 & 0.0387 \\
\hline 35 & 20 & 0.0387 & 0.0357 \\
\hline
\end{tabular}

Table 2. Effect of Training and Testing Dataset on Functional rms Error for Torque Coefficient Prediction

\begin{tabular}{|c|c|c|c|}
\hline \multicolumn{2}{|c|}{ Number of Dataset } & \multicolumn{2}{c|}{ Functional Rms Error in Prediction } \\
\hline Training Data & Testing Data & Training Error & Testing Error \\
\hline 10 & 5 & 0.0019 & 0.0860 \\
\hline 15 & 10 & 0.0390 & 0.1101 \\
\hline 20 & 13 & 0.0360 & 0.1009 \\
\hline 25 & 18 & 0.0367 & 0.0599 \\
\hline 35 & 20 & 0.0449 & 0.0283 \\
\hline
\end{tabular}

Table 1 shows the result of neural network prediction for power coefficient for different size of the training and testing datasets, while Table 2 shows the result of neural network prediction for torque coefficient for different size of the training and testing datasets. Five sets of results are shown for power and torque coefficients respectively. The numbers of training and testing dataset are kept constant for both the cases. Initially, results are obtained for 10 training data and 5 testing data. It shows high testing error, though the training error is less. In order to increase the prediction accuracy further, dataset are transferred from the testing dataset to the training dataset, and fresh data replenishes the testing dataset. In that manner, the size of training and testing datasets are increased, as shown in the respective tables. The 


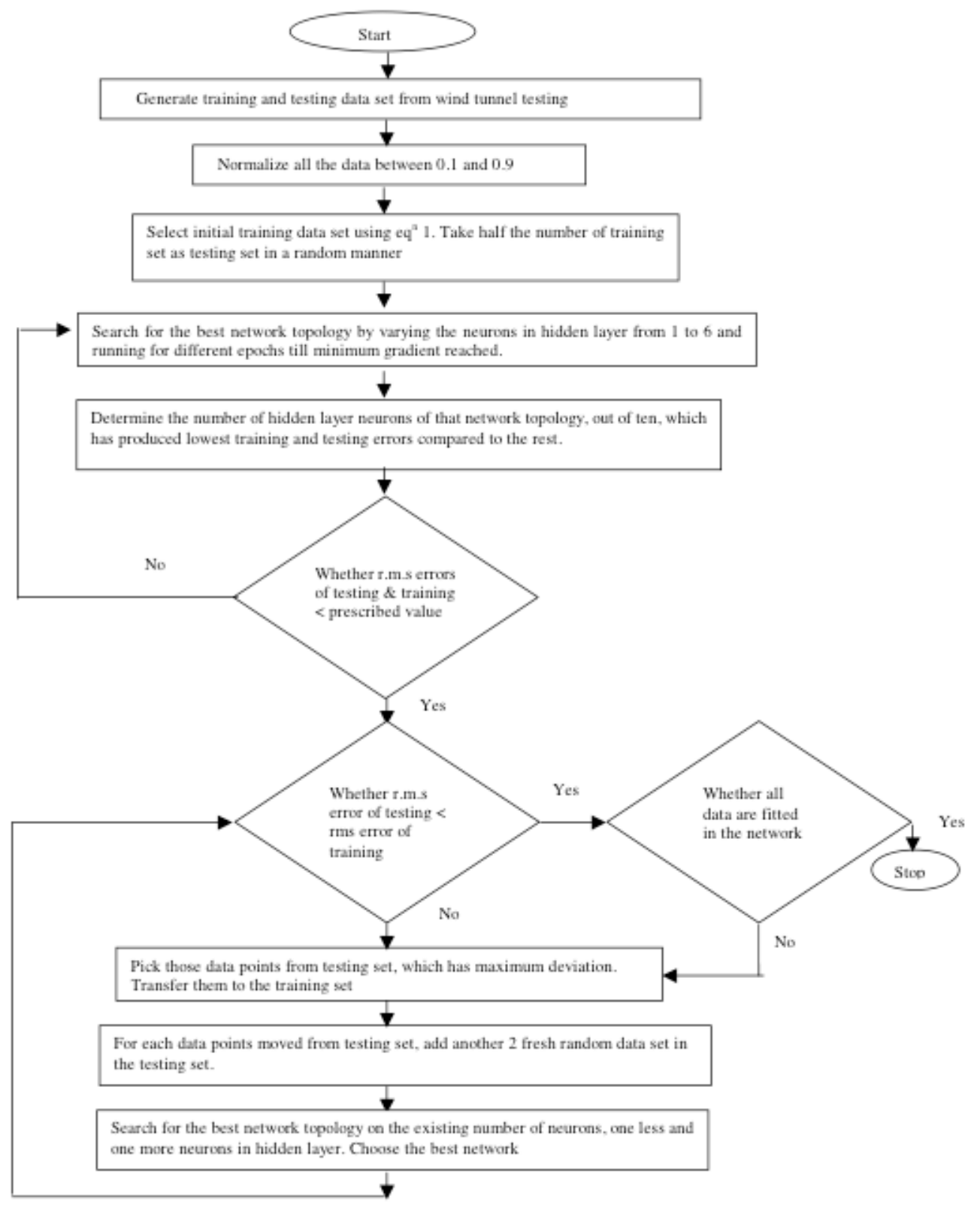

Fig. (5). The flow chart of the methodology adopted.

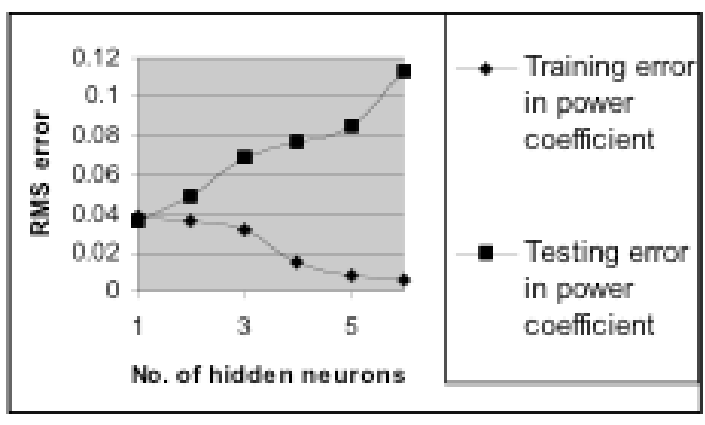

Fig. (6). The effect of hidden layer neurons on functional rms errors for power coefficient prediction.

training and testing error for the new network topologies are then compared, and the process continues till testing error is less than training error. The Table $\mathbf{1}$ and Table $\mathbf{2}$ show that, with 35 training data and 20 testing data, the desired goals are achieved. The rms error in prediction of power coefficient being slightly higher than that of torque coefficient indicates that there may have been more noise present in the data [9].

The effect of increasing hidden layer neurons on the functional $\mathrm{rms}$ errors of both training and testing for power coefficient prediction is shown in Fig. (6). Fig. (6) shows that with the increase in the number of hidden layer neurons, the rms error in training progressively decreases, while in testing, it increases. With the increase in neurons from 1 to 6 , the training error decreases from 0.0387 to 0.0060 , but the testing error increases from 0.0357 to a maximum of 0.1130 . Fig. (7) shows the effect of increasing hidden layer neurons 


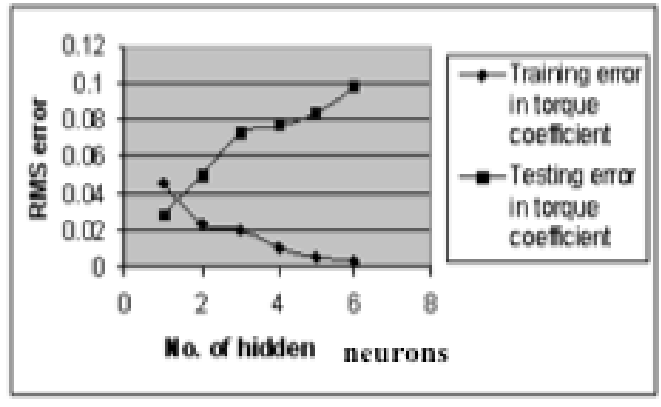

Fig. (7). The effect of hidden layer neurons on functional rms errors for torque coefficient prediction.

on the functional rms errors of both training and testing for torque coefficient prediction. In this case also, with increase of hidden layer neurons, the training error decreases from 0.0449 to 0.0036 , and the testing error increases from 0.0283 to a maximum of 0.0975 .

Fig. (8) shows the result of neural network predicted power coefficient versus target experimental power coefficient. A line inclined to $45^{\circ}$ and passing through the origin is also drawn in the same figure. For perfect prediction, all points should lie on the $45^{\circ}$ line. Here, it is seen that most of the points are close to this line. In order to get an idea about the percentage error in prediction, lines representing $\pm 5 \%$, $\pm 10 \%$ and $\pm 20 \%$ deviations are drawn. It is found from the Fig. (8) that out of 20 predictions, 7 predictions are within $\pm 5 \%$ accuracy, 14 predictions are within $\pm 10 \%, \&$ all predictions are within $\pm 20 \%$ accuracy. The maximum absolute error in prediction of power coefficient is 0.1178 . The rms errors in training and testing for prediction of power coefficient have turned out to be 0.0387 and 0.0357 respectively. Fig. (9) shows the result of neural network predicted torque coefficient versus target experimental torque coefficient. In this case also, most of the points lie close to the $45^{\circ}$ line for perfect prediction. It can be seen from Fig. (9) that out of 20 predictions, 7 predictions are within $\pm 5 \%$ accuracy, 11 predictions are within $\pm 10 \%$ and all predictions are within $\pm 20 \%$ accuracy. The maximum absolute error in prediction of torque coefficient is 0.1194 . Some of the data points are bit scattered; this is for the reason that neural network is statistical in nature. The rms errors in training and testing for prediction of torque coefficient have turned out to be 0.0449 and 0.0283 respectively.

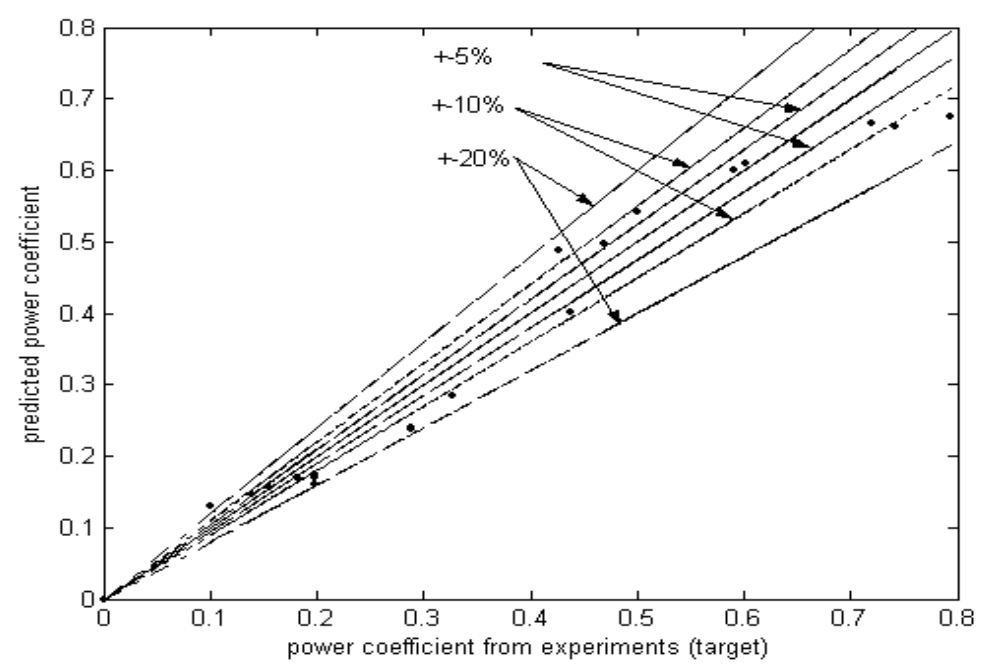

Fig. (8). Predicted power coefficient versus target power coefficient.

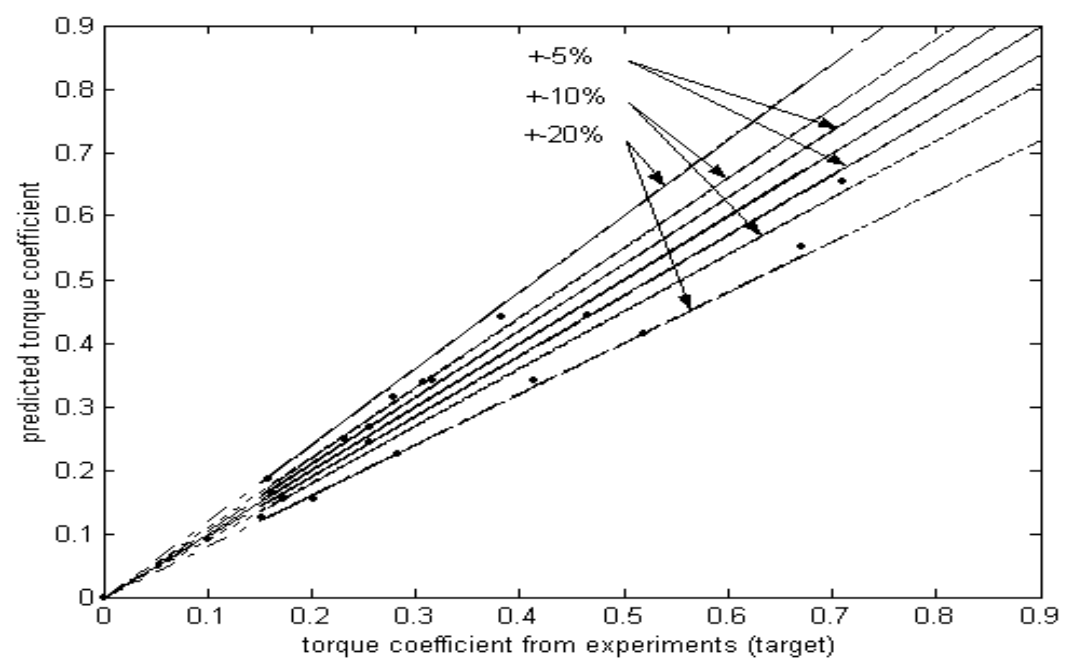

Fig. (9). Predicted torque coefficient versus target torque coefficient. 


\section{CONCLUSION}

In this paper, an attempt has been made to predict power coefficient and torque coefficient of a two bladed airfoil shaped H-rotor using ANN. A new methodology is proposed. The proposed methodology considers the fact that the ANN model can be best applied for prediction of power coefficient and torque coefficient if its prediction accuracy is high, i.e. the functional rms error of prediction is not greater than $5 \%$ in general [1]. For this, the network is initially trained with minimum training data and then tested with few testing data. Observing the accuracy in prediction, more data have been added progressively. The process is continued till the time both training and testing errors are minimized, and both brought down below the acceptable accuracy limit of $5 \%$. Twenty validation data i.e., test data have been found suitable for predicting power coefficient and torque coefficient successfully within that limit. And only a single hidden layer neuron has been utilized for prediction of both the coefficients. The root mean squared functional errors in testing and training of power coefficient are 0.0357 and 0.0387 respectively, and the corresponding errors of torque coefficient are 0.0283 and 0.0449 respectively. The proposed methodology is fast and accurate. And testing error being less than the training error, makes the proposed algorithm a superior one. Therefore, the proposed algorithm can be used for predictions of power coefficient and torque coefficient of $\mathrm{H}$-rotor type of wind rotor.

\section{Appendix}

Table A-1. Training Data Set for Power Coefficient

\begin{tabular}{|c|c|c|c|}
\hline $\begin{array}{l}\text { Blade Tip Speed } \\
\qquad(\mathrm{m} / \mathrm{s})\end{array}$ & $\begin{array}{l}\text { Free Stream Velocity With Blockage } \\
\qquad(\mathrm{m} / \mathrm{s})\end{array}$ & $\begin{array}{l}\text { Rotor Inlet Velocity } \\
(\mathbf{m} / \mathbf{s})\end{array}$ & $\begin{array}{c}\text { Power Coefficient } \\
\left(\mathbf{C}_{\mathrm{p}}\right)\end{array}$ \\
\hline 3.8227 & 28.8510 & 23.9630 & 0.0914 \\
\hline 4.8440 & 33.6860 & 30.7360 & 0.1219 \\
\hline 4.8202 & 34.4790 & 30.9360 & 0.1126 \\
\hline 4.9549 & 34.5050 & 31.4480 & 0.1193 \\
\hline 4.9788 & 34.4790 & 31.1930 & 0.1177 \\
\hline 5.2424 & 34.5580 & 32.9340 & 0.1378 \\
\hline 6.3605 & 34.5840 & 31.8250 & 0.1312 \\
\hline 6.9756 & 33.2330 & 27.3740 & 0.1424 \\
\hline 7.2918 & 33.8210 & 28.2520 & 0.1504 \\
\hline 7.4996 & 35.8320 & 29.4960 & 0.1418 \\
\hline 8.3934 & 31.2720 & 32.0430 & 0.2754 \\
\hline 7.6159 & 36.2660 & 29.6310 & 0.1435 \\
\hline 7.6437 & 35.6350 & 29.1550 & 0.1436 \\
\hline 7.9704 & 34.6840 & 28.9620 & 0.1602 \\
\hline 7.6545 & 34.5420 & 28.9630 & 0.1598 \\
\hline 7.9922 & 34.1110 & 29.2920 & 0.1729 \\
\hline 8.0903 & 35.2980 & 30.1650 & 0.1674 \\
\hline 8.8677 & 31.6140 & 30.5040 & 0.2552 \\
\hline 8.2258 & 35.1530 & 29.8190 & 0.1684 \\
\hline 11.6298 & 31.7580 & 27.1100 & 0.2669 \\
\hline 12.1773 & 34.3410 & 28.9620 & 0.2691 \\
\hline 13.1427 & 34.1990 & 28.9630 & 0.2646 \\
\hline 10.8679 & 34.7550 & 29.2360 & 0.2213 \\
\hline 12.0030 & 33.6880 & 28.8240 & 0.3380 \\
\hline 8.9094 & 27.1050 & 24.3140 & 0.2645 \\
\hline 16.6099 & 34.3890 & 28.5460 & 0.3777 \\
\hline 12.1536 & 33.3340 & 29.1010 & 0.2779 \\
\hline 13.0374 & 32.6850 & 29.0170 & 0.3828 \\
\hline 15.6233 & 32.5960 & 29.0170 & 0.3760 \\
\hline 16.5733 & 33.0080 & 28.8240 & 0.3328 \\
\hline 17.8549 & 33.9770 & 28.2640 & 0.3138 \\
\hline 15.7973 & 26.9120 & 24.3140 & 0.4428 \\
\hline 11.6415 & 33.7730 & 29.2920 & 0.4791 \\
\hline 17.2222 & 33.7360 & 28.2640 & 0.3800 \\
\hline 15.3034 & 33.4720 & 28.8240 & 0.3390 \\
\hline
\end{tabular}


Table A-2. Testing Data Set for Power Coefficient

\begin{tabular}{|c|c|c|c|}
\hline $\begin{array}{c}\text { Blade Tip Speed } \\
(\mathbf{m} / \mathbf{s})\end{array}$ & $\begin{array}{c}\text { Free Stream Velocity with Blockage } \\
(\mathbf{m} / \mathbf{s})\end{array}$ & $\begin{array}{c}\text { Rotor inlet Velocity } \\
(\mathbf{m} / \mathbf{s})\end{array}$ \\
\hline & & & $\begin{array}{c}\text { Power Coefficient } \\
\left(\mathbf{C}_{\mathbf{p}}\right)\end{array}$ \\
6.5277 & 30.1930 & 27.4600 & 0.1749 \\
6.2684 & 31.1860 & 29.4340 & 0.1747 \\
7.2273 & 31.6430 & 29.1600 & 0.1896 \\
9.2658 & 27.8085 & 22.9620 & 0.2319 \\
5.2664 & 30.8340 & 28.2960 & 0.1402 \\
5.0838 & 30.7180 & 28.2970 & 0.1405 \\
4.4135 & 31.2350 & 30.4760 & 0.1345 \\
4.0536 & 30.9200 & 30.0800 & 0.1240 \\
3.6873 & 24.6150 & 23.8430 & 0.1405 \\
4.8213 & 33.7630 & 30.6770 & 0.1179 \\
4.9059 & 33.7640 & 32.4460 & 0.1342 \\
4.4465 & 33.6860 & 29.7530 & 0.1029 \\
16.8264 & 31.8440 & 25.5940 & 0.4080 \\
15.8837 & 33.0980 & 28.9620 & 0.3674 \\
10.7988 & 33.2680 & 28.8240 & 0.2436 \\
14.6629 & 33.1440 & 29.3730 & 0.3475 \\
12.6850 & 33.6450 & 29.1000 & 0.2898 \\
11.9706 & 34.3390 & 28.8240 & 0.2556 \\
12.7121 & 33.4090 & 29.3730 & 0.2941 \\
11.3849 & 34.8590 & 28.8240 & 0.2272 \\
\hline
\end{tabular}

Table A-3. Training Data Set for Torque Coefficient

\begin{tabular}{|c|c|c|c|}
\hline $\begin{array}{l}\text { Blade Tip Speed } \\
\qquad(\mathrm{m} / \mathbf{s})\end{array}$ & $\begin{array}{l}\text { Free Stream Velocity With Blockage } \\
\qquad(\mathrm{m} / \mathrm{s})\end{array}$ & $\begin{array}{l}\text { Rotor Inlet Velocity } \\
\qquad(\mathrm{m} / \mathbf{s})\end{array}$ & $\begin{array}{l}\text { Torque Coefficient } \\
\qquad\left(\mathbf{C}_{t}\right)\end{array}$ \\
\hline 7.2918 & 33.8210 & 28.2520 & 0.6978 \\
\hline 7.4996 & 35.8320 & 29.4960 & 0.6676 \\
\hline 7.6159 & 36.2660 & 29.6310 & 0.6776 \\
\hline 6.9756 & 33.2330 & 27.3740 & 0.6694 \\
\hline 5.2664 & 30.8340 & 28.2960 & 1.0000 \\
\hline 5.0838 & 30.7180 & 28.2970 & 0.9999 \\
\hline 4.4135 & 31.2350 & 30.4760 & 0.9999 \\
\hline 4.0536 & 30.9200 & 30.0800 & 0.9999 \\
\hline 7.9922 & 34.1110 & 29.2920 & 0.6973 \\
\hline 4.9788 & 34.4790 & 31.1930 & 0.8184 \\
\hline 12.1536 & 33.3340 & 29.1010 & 0.8047 \\
\hline 11.6416 & 33.7730 & 29.2920 & 0.9105 \\
\hline 18.3657 & 32.6850 & 29.0170 & 0.8162 \\
\hline 13.0374 & 33.6450 & 29.1000 & 0.7481 \\
\hline 14.6629 & 33.1440 & 29.3730 & 0.7854 \\
\hline 4.4466 & 33.6860 & 29.7530 & 0.7801 \\
\hline 12.7121 & 33.4090 & 29.3730 & 0.7046 \\
\hline 17.8549 & 33.9770 & 28.8240 & 0.6890 \\
\hline 13.1427 & 34.1990 & 28.9630 & 0.7587 \\
\hline 4.9549 & 34.5050 & 31.4480 & 0.8306 \\
\hline 6.2684 & 31.1860 & 29.4340 & 0.8004 \\
\hline 3.6873 & 24.6150 & 23.8430 & 0.9382 \\
\hline 8.0903 & 35.2980 & 30.1650 & 0.7303 \\
\hline 4.8440 & 33.6860 & 30.7360 & 0.8434 \\
\hline 7.2273 & 31.6430 & 29.1600 & 0.8298 \\
\hline 8.9094 & 27.1050 & 24.3140 & 0.7621 \\
\hline 3.8228 & 28.8510 & 23.9630 & 0.6898 \\
\hline 11.3849 & 34.8590 & 28.8240 & 0.6818 \\
\hline 15.7973 & 26.9120 & 24.3140 & 0.7882 \\
\hline 11.6298 & 31.7580 & 27.1100 & 0.7287 \\
\hline 12.1773 & 34.3410 & 28.9620 & 0.7650 \\
\hline 8.8677 & 31.6140 & 30.5040 & 0.9098 \\
\hline 7.9704 & 34.6840 & 28.9620 & 0.7374 \\
\hline 6.5277 & 30.1930 & 27.4600 & 0.8083 \\
\hline 11.9706 & 34.3390 & 28.8240 & 0.7730 \\
\hline
\end{tabular}


Table A-4. Testing Data Set for Torque Coefficient

\begin{tabular}{|c|c|c|c|}
\hline $\begin{array}{l}\text { Blade Tip Speed } \\
\qquad(\mathbf{m} / \mathbf{s})\end{array}$ & $\begin{array}{l}\text { Free Stream Velocity With Blockage } \\
\qquad(\mathrm{m} / \mathrm{s})\end{array}$ & $\begin{array}{l}\text { Rotor Inlet Velocity } \\
(\mathbf{m} / \mathbf{s})\end{array}$ & $\begin{array}{c}\text { Torque Coefficient } \\
\left(\mathbf{C}_{\mathrm{t}}\right)\end{array}$ \\
\hline 7.6437 & 35.6350 & 29.1550 & 0.6787 \\
\hline 8.2258 & 35.1530 & 29.8190 & 0.7195 \\
\hline 16.6099 & 34.3890 & 28.2640 & 0.7000 \\
\hline 15.3034 & 33.4720 & 28.5460 & 0.7416 \\
\hline 16.5733 & 33.0080 & 28.8240 & 0.7626 \\
\hline 4.8214 & 33.7630 & 30.6770 & 0.8255 \\
\hline 8.3934 & 31.2720 & 32.0430 & 1.0000 \\
\hline 7.6545 & 34.5420 & 28.9630 & 0.7030 \\
\hline 5.2424 & 34.5580 & 32.9340 & 0.9082 \\
\hline 9.2658 & 27.8085 & 22.9620 & 0.7100 \\
\hline 10.8679 & 34.7550 & 29.2360 & 0.7077 \\
\hline 4.8202 & 34.4790 & 30.9360 & 0.8050 \\
\hline 5.3605 & 34.5840 & 31.8250 & 0.8468 \\
\hline 4.9059 & 33.7640 & 32.4460 & 0.9234 \\
\hline 15.6233 & 32.5960 & 29.0170 & 0.7925 \\
\hline 17.2222 & 33.7360 & 28.2640 & 0.7019 \\
\hline 12.0030 & 33.6880 & 28.8240 & 0.7321 \\
\hline 15.3034 & 33.4720 & 28.8240 & 0.7416 \\
\hline 10.7988 & 33.2680 & 28.8240 & 0.7507 \\
\hline 15.8837 & 33.0980 & 28.9620 & 0.7657 \\
\hline
\end{tabular}

\section{REFERENCES}

[1] Dixit, U.S; Chandra, S. A neural network based methodology for the prediction of roll force and roll torque in fuzzy form for cold flat rolling process. Int. J. Adv. Manuf. Technol., 2003, 22, 883889.

[2] Biswas, A.; Gupta R. Comparative study of a two-bladed airfoil shaped H-Darrieus rotor with a two straight bladed H-Darrieus rotor. In Proceedings of World Renewable Energy Congress (WREC), Glasgow, Scot Land, 2008.

[3] Kalogirou, S.A. Applications of neural networks for energy systems. Renewable Energy, 2005, 30 (7), 1075-1090.

[4] Kalogirou, S.A. Artificial neural networks in renewable energy systems applications: a review. Renewable Sustain. Energy Rev., 2001, 5 (4), 373-401.
[5] Cam, E.; Arcaklioglu, E.; Cavusoglu, A.; Akbiyik, B.A. Classification mechanism for determining average wind speed and power in several regions of Turkey using artificial neural networks. Renewable Energy, 2005, 30 (2), 227-239.

[6] Gupta, R.; Das, R.; Sharma K.K. Experimental study of a SavoniusDarrieus wind machine. In Proceedings of the International Conference on Renewable Energy for Developing Countries (ICREDC), University of California, Washington DC, 2006.

[7] Eberhart. Computational intelligence PC tools. In Proceedings of AP professional, New York, 1996.

[8] Lawrence, M.; Petterson, A. Brain maker user's manual. California scientific Software, $7^{\text {th }}$ ed. Nevada City, California 2002.

[9] Kohli, A.; Dixit, U.S. A neural-network-based methodology for the prediction of surface in turning process. Int. J. Adv. Manuf. Technol., 2005, 25, 118-129.

(c) Biswas and Gupta; Licensee Bentham Open.

This is an open access article licensed under the terms of the Creative Commons Attribution Non-Commercial License (http://creativecommons.org/licenses/by-nc/3.0/) which permits unrestricted, non-commercial use, distribution and reproduction in any medium, provided the work is properly cited. 